\title{
(1)
}

\section{Evaluación de ocho líneas de arveja arbustiva (Pisum sativum L.) en seis ambientes de la zona cerealista de Nariño}

\section{Evaluation of eight shrub pea lines (Pisum sativum L.) in six environment of the Nariño cerealist zone}

\author{
Oscar Eduardo Checa Coral ${ }^{1}$; Jeferson Andrés Getial Pantoja ${ }^{2}$; Daniel Marino Rodríguez Rodríguez ${ }^{3}$
}

\begin{abstract}
II.A. Ph.D. Universidad de Nariño, sede Vipri, Facultad de Ciencias Agrícolas, Grupo de Investigación en Cultivos Andinos GRICAND. San Juan de Pasto Nariño, Colombia; e-mail: cicagrarias@hotmail.com; (D) https://orcid.org/0000-0002-6929-7717

${ }^{2}$ Estudiante de Ingeniería Agronómica. Universidad de Nariño, sede Vipri, Facultad de Ciencias Agrícolas. San Juan de Pasto - Nariño, Colombia; e-mail: getialjeferson@gmail.com; (D) https://orcid.org/0000-0001-5514-1706

${ }^{3}$ I.A. M.Sc. Universidad de Nariño, sede Vipri, Facultad de Ciencias Agrícolas, Grupo de Investigación en Cultivos Andinos GRICAND. San Juan de Pasto Nariño, Colombia; e-mail: danmaro25@hotmail.com; (D) https://orcid.org/0000-0001-9152-5897
\end{abstract}

\begin{abstract}
Cómo citar: Checa Coral, O.E.; Getial Pantoja, J.A.; Rodríguez Rodríguez, D.M. 2020. Evaluación de ocho líneas de arveja arbustiva (Pisum sativum L.) en seis ambientes de la zona cerealista de Nariño. Rev. U.D.C.A Act. \& Div. Cient. 23(1):e1211. http:// doi.org/10.31910/rudca.v23.n1.2020.1211

Artículo de acceso abierto publicado por Revista U.D.C.A Actualidad \& Divulgación Científica, bajo una licencia Creative Commons CC BY-NC 4.0

Publicación oficial de la Universidad de Ciencias Aplicadas y Ambientales U.D.C.A. Institución de Educación Superior Acreditada de Alta Calidad por el Ministerio de Educación Nacional.

Recibido: Mayo 5 de 2019

Aceptado Marzo 20 de 2020

Editado por: Ingeborg Zenner de Polanía
\end{abstract}

\section{RESUMEN}

La arveja arbustiva tiene características potenciales para la agroindustria y comercio en fresco y puede ser una alternativa para la zona cerealista de Nariño. La presente investigación, se realizó en dos municipios (Yacuanquer y Guaitarilla), pertenecientes a la zona cerealista de Nariño, durante tres épocas de siembra, en el 2017. La combinación de localidades por épocas constituyeron los seis ambientes estudiados. Se evaluaron ocho líneas de arveja arbustiva por número de vainas por planta, adaptabilidad y estabilidad fenotípica, para rendimiento en vaina verde y reacción a enfermedades foliares. En número de vainas sobresalieron GRICAND404 y GRICAND402. El análisis de adaptabilidad y de estabilidad fenotípica permitió identificar, como ambientes favorables, a Yacuanquer abril, Guaitarilla abril y Yacuanquer marzo;
GRICAND405, con un rendimiento promedio de 9,27t.ha ${ }^{-1}$, se consideró una línea adaptable y predecible en los seis ambientes evaluados y GRICAND406, con 9,07t.ha ${ }^{-1}$, fue predecible y mostró mejor adaptación a los ambientes más favorables. En reacción a las enfermedades evaluadas, todas las líneas fueron susceptibles al ataque del complejo Ascochyta y el porcentaje de infestación de la enfermedad, se evidenció mayor en épocas muy lluviosas, como la época 1 (siembra de marzo) y disminuyó en épocas secas, como la época 3 (siembra de mayo); el ataque de Oídio, se evidenció mayormente en la época de menor precipitación (época 3), siendo resistente la línea GRICAND402.

Palabras clave: líneas; condiciones atmosféricas; adaptabilidad; estabilidad genética; reacción a enfermedades. 


\section{ABSTRACT}

The shrub pea has potential characteristics for agroindustry and fresh trade and can be an alternative for the cereal zone of Nariño. The present investigation was carried out in two municipalities (Yacuanquer and Guaitarilla) belonging to the Nariño cereal zone during three sowing seasons in 2.017; the combination of the localities by the times was the six environments studied. Eight shrub pea lines were evaluated by number of pods per plant, adaptability and phenotypic stability to yield in green pod and reaction to foliar diseases. The number of pods stood out GRICAND404 and GRICAND402. The analysis of adaptability and phenotypic stability made it possible to identify the favorable environments for Yacuanquer april, Guaitarilla april and Yacuanquer march; GRICAND405 with an average yield of 9.27t.ha-1 was considered the adaptable and predictable line in the six environments evaluated and GRICAND406 with 9.07t.ha-1 was predictable and showed better adaptation to the most favorable environments. In reaction to diseases, all the lines were susceptible to attack by the Ascochyta complex and the percentage of infestation of the disease was greater in very rainy seasons such as season 1 (march sowing) and decreased in dry seasons such as season 3 (planting of may), the attack of Oídio was evidenced mainly in the period of least precipitation (season 3), being resistant the GRICAND402 line.

Keywords: lines; meteorological factors; adaptability; genetic stability; reactions to diseases.

\section{INTRODUCCIÓN}

La arveja (Pisum sativum L.) es una especie diploide extendida por todo el mundo, debido a su amplia diversidad genética, que permite su desarrollo en diferentes regiones y climas. Es originaria de una extensa área, que incluye Asia central, el cercano Oriente, Etiopía y el Mediterráneo, siendo una de las 15 principales leguminosas alimenticias, ya sea en grano fresco, grano seco o en productos procesados como harinas y conservas (Bolaños, 2001).

La arveja ha sido un factor estabilizador de la economía de los pequeños productores de las zonas andinas y ha contribuido a su seguridad alimentaria. Se estima que de este cultivo involucra más de 26.000 productores, generando cerca de 2,3 millones de jornales (Camargo \& Ávila, 2014). Según Fenalce (2016), en el primer semestre del 2016, se sembraron 16.493 hectáreas de arveja, arrojando una producción de 83.800 toneladas en vaina verde. En Colombia, el departamento de Nariño es principal productor, con 32.900 toneladas, seguido por los departamentos de Cundinamarca, Boyacá y Tolima. Las variedades más utilizadas en Nariño son Sureña, San Isidro, Obonuco Andina y Sindamanoy, siendo todas de hábito de crecimiento voluble (Checa \& Rodríguez, 2015). No se cuenta, hasta el momento, con arvejas de hábito de crecimiento arbustivo.

En Nariño, la zona dedicada a los cereales menores que se ubica entre los 2.000 y $2.800 \mathrm{~m}$ s.n.m. no presenta opciones de rotación para mejorar los ingresos del productor (Checa et al. 2017), por lo cual, se considera que encontrar materiales de arvejas arbustivas adaptados a la zona con una adecuada época de siembra podrían ser una alternativa productiva, permitiendo al productor participar en el mercado fresco de esta especie con un buen margen de rentabilidad; asimismo, la producción en seco puede contribuir, en parte, a la sustitución de importaciones que se realizan para satisfacer la demanda nacional.

La evaluación de genotipos, a través de diferentes localidades y semestres, es una importante opción para estimar las respuestas genotípicas diferenciales a distintas condiciones ambientales y determinar la interacción genotipo-ambiente, además permite evaluar los potenciales productivos y las posibles limitaciones de éstos en cada localidad (Aulicino et al. 2000; Mora et al. 2007). De acuerdo con González \& Ligarreto (2006), la temperatura y la precipitación son factores altamente influyentes en la producción y desarrollo de la arveja, con grandes efectos sobre el rendimiento y sus componentes.

En este sentido, la presente investigación se realizó, con el fin de contribuir a la búsqueda de una nueva alternativa productiva para la zona cerealista de Nariño, a través de la evaluación de ocho genotipos de arveja arbustiva (Pisum sativum L.), en los municipios de Yacuanquer y Guaitarilla.

\section{MATERIALES Y MÉTODOS}

Localización: La investigación, se llevó a cabo entre marzo y octubre de 2017, en dos municipios de la zona cerealista de Nariño, correspondientes a la localidad de Yacuanquer, ubicada a $01^{\circ} 07^{\prime} 32,9^{\prime \prime} \mathrm{N}$ y $77^{\circ} 24^{\prime} 04,6^{\prime \prime} \mathrm{W}$, altura $2.744 \mathrm{~m}$ s.n.m., temperatura promedio $13,1^{\circ} \mathrm{C}$ y precipitación anual de $1.075 \mathrm{~mm}$ y Guaitarilla, ubicada a $01^{\circ} 06^{\prime} 49,4^{\prime \prime} \mathrm{N}, 77^{\circ} 32^{\prime} 02,4^{\prime \prime} \mathrm{W}$, altura $2.825 \mathrm{~m}$ s.n.m., temperatura promedio $13,5^{\circ} \mathrm{C}$ y precipitación anual de $1.303 \mathrm{~mm}$. En ambas localidades, el brillo solar de 4 a 5 horas en suelos Pachic melanudands (IGAC, 2004).

Diseño experimental: La evaluación, se realizó en dos localidades y tres épocas de siembra, para un total de seis ensayos. En cada ensayo, se utilizó un diseño de bloques al azar con ocho tratamientos que correspondieron a las ocho líneas a evaluar y cuatro repeticiones. Las localidades fueron Yacuanquer y Guaitarilla, las épocas de siembra fueron: época 1, siembra en marzo; época 2, siembra en abril y época 3, siembra en mayo. La combinación de las dos localidades por las tres épocas de siembra generó los seis ambientes evaluados, denominados Yacuanquer marzo, Yacuanquer abril, Yacuanquer mayo, Guaitarilla marzo, Guaitarilla abril y Guaitarilla mayo.

Las ocho líneas de arveja arbustiva evaluadas pertenecen a la colección de trabajo en mejoramiento genético de esta leguminosa, desarrollado por el Grupo de Investigación en Cultivos Andinos GRICAND, de la Facultad de Ciencias Agrícolas de la Universidad de Nariño. La identificación y genealogía de las líneas es la siguiente: GRICAND401 (ILS3585 SI Nikado Obonuco Col.), GRICAND402 (UN6651 1205-MA-M), GRICAND403 (ILS3572 SI Amino INTA Argentina), GRICAND404 (ILS3566 SI Brasil 7), GRICAND405 (Sx3575 RC1-F2-20-P1), GRICAND406 (SIxD RC1-F2-L100- 
SI-P3), GRICAND407 (Sx3575-F6-L1-SI-P5) y GRICAND408 (Sx3575-F6-1-SI-P3). Las cuatro primeras presentan hojas normales y las cuatro restantes hojas afilas, cuya expresión fenotípica es el remplazo de hojas laterales por zarcillos, carácter que es conferido por el gen recesivo of (Kujala, 1953; Hedley \& Ambrose, 1981; Prohens \& Nuez, 2007).

La unidad experimental estuvo conformada por seis surcos de tres metros de largo, con distancia entre surcos de $0,5 \mathrm{~m}$ y entre sitios de $0,1 \mathrm{~m}$, ubicando una semilla por sitio, para obtener 150.000 plantas. $\mathrm{ha}^{-1}$. Para la evaluación, se descartaron los surcos borde, por tanto, la parcela útil correspondió a los cuatro surcos centrales. De las cuatro repeticiones, en tres, se evaluaron las variables planteadas y, en la última repetición de cada experimento, se evaluó la reacción a enfermedades foliares (complejo Ascochyta y Oídio), evitando la aplicación de control químico.

La variable precipitación mensual que sirvió para contrastar con la respuesta obtenida de las líneas en cada época fue facilitada por el servicio de información del IDEAM.

Variables evaluadas: Número de vainas por planta (NVP): se contabilizó el total de vainas de la parcela útil y se dividió por el número de plantas cuantificadas; rendimiento en vaina verde (RTOVV): se obtuvo el peso de las vainas de los surcos de la unidad experimental llevándolo a t.ha ${ }^{-1}$.

La reacción a enfermedades, como el complejo Ascochyta (Ascochyta pisi y Mycosphaerella pinodes) y Oídio (Erisyphe pisi), se hizo en la última repetición de cada ensayo, en la cual, los patógenos foliares no fueron controlados. Esta evaluación, se realizó con base en las escalas utilizadas por Valencia et al. (2012), para el complejo Ascochyta y Checa \& Rodríguez (2015), para Oídio.

Análisis estadístico: Con los datos obtenidos, se realizó un análisis de varianza combinado, utilizando, como efectos fijos, las Líneas y, como efectos aleatorios, los factores Localidades y Épocas. Para las variables que mostraron significancia en el Andeva, se llevaron a cabo las pruebas de comparación de promedios de Tukey $(P<0,05)$.

Análisis de adaptabilidad y estabilidad fenotípica: Se aplicó el modelo propuesto por Eberhart \& Russell (1966), basado en la media, la regresión lineal y las desviaciones de la regresión. Este método ha sido muy utilizado en diferentes investigaciones, para determinar la adaptabilidad y la estabilidad de genotipos, a través de distintos ambientes (Ligarreto et al. 2015; Vargas et al. 2016; Vásques et al. 2019). La variable analizada fue el rendimiento en vaina verde para ocho líneas y seis ambientes, resultantes de la combinación de las dos localidades (Yacuanquer y Guaitarilla), por las tres épocas de siembra (marzo, abril y mayo).

\section{RESULTADOS Y DISCUSIÓN}

Para las siembras realizadas en el ambiente de Yacuanquer marzo (Yac Mar), el acumulado de precipitación durante todo el ciclo de cultivo fue de 560,7mm; para Yacuanquer abril (Yac Abr), de 404mm y Yacuanquer mayo (Yac May), de 313,9mm. Un comportamiento similar se observó en los otros ambientes, donde para Guaitarilla marzo (Gua Mar), el acumulado fue de 626,5mm; Guaitarilla abril (Gua Abr), de 423mm y Guaitarilla Mayo (Gua May), de 327,7mm. El promedio de precipitación fue variable en todos los ambientes, así: los ambientes de la siembra realizada en marzo fueron los que presentaron mayor precipitación en el primer y tercer mes del ciclo; los ambientes de la siembra de abril presentaron lluvias moderadas con la máxima precipitación en el segundo mes y los de la siembra de mayo tuvieron una alta precipitación en el primer mes y posteriormente mostraron una fuerte reducción de la lluvia, siendo la época más seca de las tres evaluadas (Figura 1) (IDEAM, 2018).

Análisis de varianza: $\mathrm{El}$ análisis de varianza combinado para las variables NVP y RTOVV mostró diferencias significativas para Líneas y para las interacciones dobles Localidad*Época, Líneas*Localidad, Líneas*Época. De igual manera, fue significativa la interacción triple Líneas*Localidad*Época. No se obtuvo diferencias significativas para los efectos simples de localidad y época (Tabla 1).

Número de vainas por planta (NVP): En la tabla 2, se observa, para la localidad de Yacuanquer, en la época 1, que la línea GRICAND404, con 20,33 vainas, supera a las demás, exceptuando a GRICAND407 y GRICAND402, que lograron promedios de 17,66 y 17,33 vainas respectivamente; las demás líneas obtuvieron entre 13 y 16,66 vainas por planta. En la época 2, GRICAND402, con 20 vainas por planta, supera a GRICAND408, GRICAND406, GRICAND404 y GRICAND405, con promedios de 13,66 y 16 vainas. Para la época 3, GRICAND401 y GRICAND404, con 12,33 y 11,66 vainas, respectivamente, sobresalen sobre el resto de las líneas, las cuales, estuvieron entre 5,33 y 7,66 vainas por planta, exceptuando a GRICAND402, con 10 vainas.

En la localidad de Guaitarilla, en la época 1, se observó que GRICAND404 y GRICAND405, con 15 vainas por planta, superaron al resto de las líneas, que obtuvieron entre 8,33 y 11 vainas. Para la época 2, GRICAND404, con 19,33 vainas, superó a todas las líneas, excepto a GRICAND401 y GRICAND405 (18 y 16,66 vainas, respectivamente). En la época 3, GRICAND401, GRICAND404 y GRICAND402, con promedios desde 11 a 11,66 vainas por planta, superaron a GRICAND406 y GRICAND407, con 7,33 y 7 vainas, respectivamente; las demás líneas obtuvieron promedios de 8,33 a 10 vainas por planta.

Yacuanquer época 1 (15,95 NVP), época 2 (16,58 NVP) y Guaitarilla época $2(15,20 \mathrm{NVP})$ presentaron los mejores promedios en número de vainas por planta, mostrando una relación directa con la variación climática, debido a la buena distribución de las lluvias y condiciones edáficas presentadas en el lugar de la investigación; en estos ambientes sobresalieron las líneas GRICAND404, con 20,33 y 19,33 vainas por planta y GRICAND402, con 20 vainas. En contraste, en las épocas de baja intensidad de lluvias (época 3), se observó una disminución en el número de vainas por planta, debido a la deficiente oferta hídrica durante el periodo de cultivo; en esta época, se destacan las líneas GRICAND401, GRICAND402 y GRICAND404, con promedios desde 10 a 12,33 vainas por planta. $\mathrm{Al}$ respecto, Sañudo 
Tabla 1. Cuadrados medios para componentes de rendimiento en la evaluación de 8 líneas de arveja arbustiva, a través de 2 localidades y 3 épocas de siembra, en la zona cerealista de Nariño.

\begin{tabular}{|c|c|c|c|}
\hline F.V & GL & NVP & RTOVV \\
\hline Localidad & 1 & 110,25 & 51,21 \\
\hline Época & 2 & 602,92 & 315,99 \\
\hline Localidad*Época & 2 & $110,77^{* *}$ & $149,76^{* *}$ \\
\hline Error A & 12 & 1,03 & 1,28 \\
\hline Bloques en 6 exptos. & 17 & 824,97 & 518,24 \\
\hline Líneas & 7 & $50,15^{* *}$ & $41,74^{* *}$ \\
\hline Líneas*Localidad & 7 & $15,45^{* *}$ & $12,17 * *$ \\
\hline Líneas*Época & 14 & $12,55^{* *}$ & $9,22^{* *}$ \\
\hline Líneas*Localidad*Época & 14 & $6,95^{* *}$ & $7,80^{* *}$ \\
\hline Error B & 84 & 1,14 & 1,16 \\
\hline CV & & 8,36 & 12,76 \\
\hline
\end{tabular}

F.V: Fuentes de variación; GL: grados de libertad; CV: coeficiente de variación; NVP: número de vainas por planta; RTOVV: rendimiento vaina verde; * = significancia a nivel del 0,$05 ; *=$ significancia a nivel del 0,01 .

Tabla 2. Comparación de promedios de Tukey en la variable NVP para 8 líneas de arveja arbustiva, a través de 2 localidades y 3 épocas de siembra, en la zona cerealista de Nariño.

\begin{tabular}{|l|c|c|c|c|c|c|}
\hline \multicolumn{7}{|c|}{ Yocalidades } \\
\hline Líneas & Época 1 & Época 2 & Época 3 & Época 1 & Época 2 & Época 3 \\
\hline GRICAND401 & $13,00 \mathrm{e}$ & $17,33 \mathrm{ab}$ & $12,33 \mathrm{a}$ & $11,00 \mathrm{~b}$ & $18,00 \mathrm{ab}$ & $11,66 \mathrm{a}$ \\
\hline GRICAND402 & $17,33 \mathrm{abc}$ & $20,00 \mathrm{a}$ & $10,00 \mathrm{ab}$ & $8,33 \mathrm{~b}$ & $15,66 \mathrm{bcd}$ & $11,00 \mathrm{a}$ \\
\hline GRICAND403 & $14,00 \mathrm{cde}$ & $18,33 \mathrm{ab}$ & $7,66 \mathrm{bc}$ & $8,66 \mathrm{~b}$ & $12,00 \mathrm{e}$ & $8,33 \mathrm{ab}$ \\
\hline GRICAND404 & $20,33 \mathrm{a}$ & $15,33 \mathrm{bc}$ & $11,66 \mathrm{a}$ & $15,00 \mathrm{a}$ & $19,33 \mathrm{a}$ & $11,66 \mathrm{a}$ \\
\hline GRICAND405 & $16,66 \mathrm{bcd}$ & $16,00 \mathrm{bc}$ & $7,00 \mathrm{cb}$ & $15,00 \mathrm{a}$ & $16,66 \mathrm{abc}$ & $10,00 \mathrm{ab}$ \\
\hline GRICAND406 & $15,33 \mathrm{bcde}$ & $15,00 \mathrm{bc}$ & $6,33 \mathrm{cde}$ & $10,33 \mathrm{~b}$ & $12,66 \mathrm{de}$ & $7,33 \mathrm{~b}$ \\
\hline GRICAND407 & $17,66 \mathrm{ab}$ & $17,00 \mathrm{abc}$ & $6,66 \mathrm{bc}$ & $10,66 \mathrm{~b}$ & $13,00 \mathrm{de}$ & $7,00 \mathrm{~b}$ \\
\hline GRICAND408 & $13,33 \mathrm{de}$ & $13,66 \mathrm{c}$ & $5,33 \mathrm{c}$ & $9,00 \mathrm{~b}$ & $14,33 \mathrm{cde}$ & $8,66 \mathrm{ab}$ \\
\hline Promedio & $15,95 \mathrm{ab}$ & $16,58 \mathrm{a}$ & $8,37 \mathrm{e}$ & $10,99 \mathrm{c}$ & $15,20 \mathrm{~b}$ & $9,45 \mathrm{~d}$ \\
\hline
\end{tabular}

Promedios con las mismas letras no son significativamente diferentes.

et al. (1999) afirman que, condiciones secas en las fases de desarrollo vegetativo y floración, conducen a reducción en el número de vainas. También, hubo una disminución del número de vainas en la época 1 en Guaitarilla, debido al exceso de precipitación entre el primer y tercer mes del cultivo (Figura 1), que comprende las fases iniciales de crecimiento hasta floración. Los resultados encontrados concuerdan con los reportados por González \& Ligarreto (2006), Pacheco et al. (2011) y Casanova et al. (2012), quienes obtuvieron valores entre 20,25 y 14,50 vainas por planta. En contraste, Siddique et al. (2002) reportaron valores bajos en genotipos arbustivos con 6,8 vainas por planta. Summerfield et al. (1991) concluyeron que, además del genotipo, hay factores ambientales externos, que influyen sobre el crecimiento y el desarrollo de las plantas, como la temperatura, el fotoperiodo y la disponibilidad de agua y nutrientes.
Adaptabilidad y estabilidad fenotípica para rendimiento en vaina verde: La significativa interacción Líneas*Localidad*Época, obtenida en el análisis de varianza combinado, permitió realizar el análisis de adaptabilidad y de estabilidad, propuesto por Eberhart \& Russel (1966). Los índices ambientales (Tabla 3) indican que los ambientes más favorables fueron Yacuanquer abril, Guaitarilla abril y Yacuanquer marzo y los menos favorables Guaitarilla mayo, Guaitarilla marzo y Yacuanquer mayo. Lo anterior concuerda con lo que se deriva de las condiciones de precipitación que se presentaron en los diferentes ambientes y épocas de siembra (Figura 1), en donde la mejor época para la siembra corresponde a abril, tanto para Yacuanquer como para Guaitarilla. En contraste, la excesiva humedad que se presentó en marzo, afectó más a la localidad de Guaitarilla que a Yacuanquer que, a pesar del exceso de humedad, 
logró altos rendimientos, por contar con un drenaje apropiado. Finalmente, entre los ambientes de menor respuesta de rendimiento está la siembra realizada en mayo, la cual, estuvo caracterizada por una drástica reducción en la precipitación (Figura 1).
Observando el desempeño en rendimiento de las líneas evaluadas, se pudo notar que las cuatro líneas con gen afila (GRICAND405, GRICAND406, GRICAND407 y GRICAND408), con promedios entre 8,52 y 9,92, superaron a la media general; no así, las líneas

Tabla 3. Rendimiento promedio e índices ambientales y parámetros en el análisis de adaptabilidad y de estabilidad fenotípica, para producción en vaina verde $\left(\mathrm{t} \cdot \mathrm{ha}^{-1}\right)$.

\begin{tabular}{|c|c|c|c|}
\hline \multicolumn{2}{|c|}{ Ambientes } & $\operatorname{Media}\left(\mathrm{t} . \mathrm{ha}^{-1}\right)$ & Índices ambientales \\
\hline \multicolumn{2}{|l|}{ Yacuanquer Abril } & 11,43 & 2,99 \\
\hline \multicolumn{2}{|l|}{ Guaitarilla Abril } & 10,94 & 2,50 \\
\hline \multicolumn{2}{|l|}{ Yacuanquer Marzo } & 10,54 & 2,10 \\
\hline \multicolumn{2}{|l|}{ Guaitarilla Mayo } & 7,07 & $-1,37$ \\
\hline \multicolumn{2}{|l|}{ Guaitarilla Marzo } & 5,52 & $-2,92$ \\
\hline \multicolumn{2}{|l|}{ Yacuanquer Mayo } & 5,14 & $-3,30$ \\
\hline Líneas & $\operatorname{Media}\left(\right.$ t.ha $\left.{ }^{-1}\right)$ & Beta $=1$ & $\mathrm{~S}^{2}=0$ \\
\hline GRICAND401 & 5,64 & $0,25 * *$ & $3,11 * *$ \\
\hline GRICAND402 & 9,60 & $1,49 * *$ & $1,52 * *$ \\
\hline GRICAND403 & 6,56 & $0,88 \mathbf{n s}$ & $1,91 * *$ \\
\hline GRICAND404 & 8,93 & $0,74 * *$ & $1,87 * *$ \\
\hline GRICAND405 & 9,27 & $0,98 \mathbf{n s}$ & $0,56 \mathbf{n s}$ \\
\hline GRICAND406 & 9,07 & $1,28 * *$ & $0,49 \mathbf{n s}$ \\
\hline GRICAND407 & 9,92 & $1,32 * *$ & $2,72 * *$ \\
\hline GRICAND408 & 8,52 & $1,06 \mathbf{n s}$ & $0,76 *$ \\
\hline Media general & 8,44 & & \\
\hline
\end{tabular}

$*=$ significancia a nivel del 0,$05 ; * *=$ significancia a nivel del 0,$01 ;$ ns: diferencias no significativas.

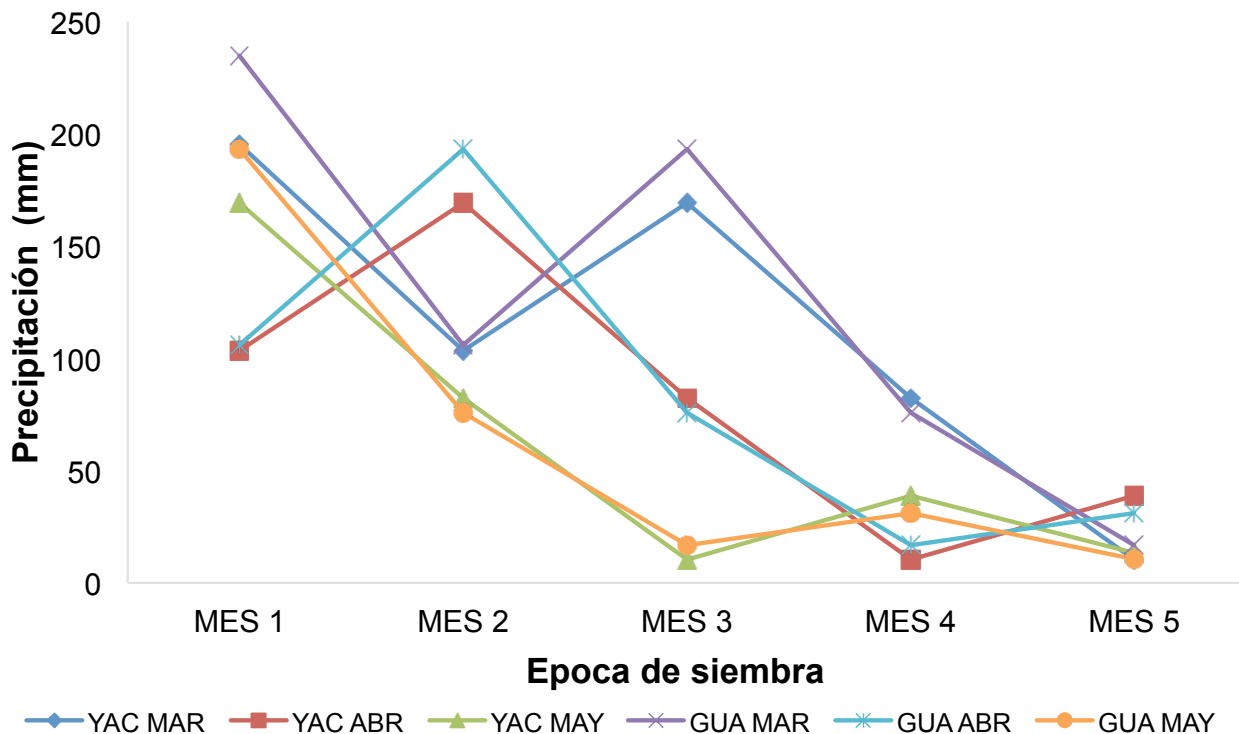

Figura 1. Precipitación para Yacuanquer y Guaitarilla en tres épocas de siembra. Fuente: IDEAM, 2018. 
con hojas normales, en donde dos de ellas (GRICAND401 y GRICAND403), estuvieron por debajo del promedio, que fue 8,44 . Estos resultados sugieren que la presencia del gen afila no es causante de reducción del rendimiento y confirman las observaciones de Wang et al. (2003), Jannink et al. (1996) y Mihailovic (2008), quienes reportaron que el mayor desarrollo de zarcillos que se presentan en variedades afilas contribuyen a la resistencia al volcamiento y pueden incrementar su productividad respecto a las variedades no afila.

Los parámetros de adaptabilidad y de estabilidad fenotípica indican que la línea GRICAND405, con valores de $\beta=1$ y $\mathrm{S}^{2}=0$ (Tabla 3, Figura 2), mostró la mejor adaptación a las diferentes condiciones ambientales, con un comportamiento predecible; además, esta línea, con una media de $9,27 \mathrm{t} \mathrm{ha}^{-1}$, superó a la media general, constituyéndose en una interesante opción productiva de arveja en vaina verde, para las condiciones edafoclimáticas de los ambientes evaluados, en la zona cerealista de Nariño.

Las líneas GRICAND403, con un rendimiento de 6,56t.ha ${ }^{-1}$, inferior a la media general y GRICAND408, con 8,52t.ha ${ }^{-1}$, superior a la media general, presentaron un $\beta=1$, que indica que fueron adaptables a los distintos ambientes, pero los desvíos de la regresión $\mathrm{S}^{2}$ fueron significativamente mayores de cero (Tabla 3, Figura 2), que implica que estos dos genotipos no fueron predecibles $\mathrm{y}$, en consecuencia, no es posible asegurar el mismo comportamiento

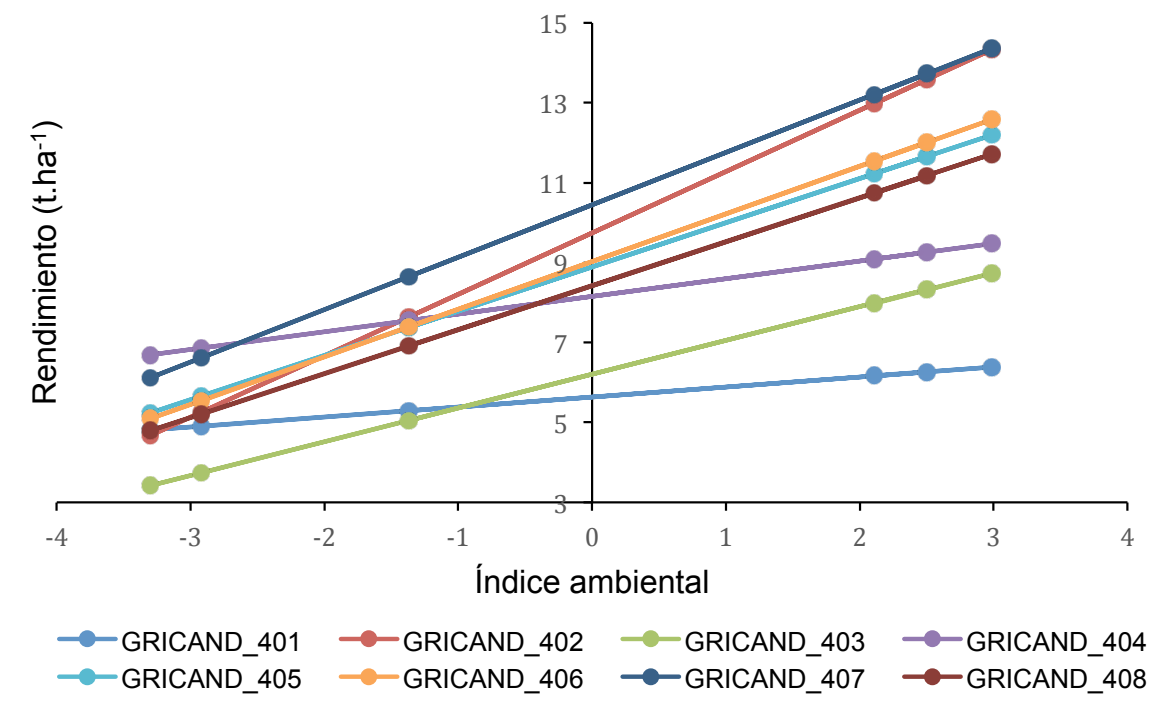

Figura 2. Adaptabilidad de ocho líneas de arveja arbustiva, en seis ambientes de la zona cerealista de Nariño.

agronómico para rendimiento en futuras siembras que permita su selección, en especial, para GRICAND408, que mostró alto rendimiento promedio.

GRICAND406, con una media de 9,07t.ha ${ }^{-1}$, que estuvo por encima de la media general, presentó un $\beta$ significativamente superior a 1 , indicando que tiene mejor respuesta a ambientes favorables, como Yacuanquer abril, Guaitarilla abril y Yacuanquer marzo; además, presentó un comportamiento predecible $\left(\mathrm{S}^{2}=0\right.$ ) (Tabla 3, Figura 2). En consecuencia, esta línea se puede recomendar como una buena alternativa de producción para las siembras de abril, en los municipios mencionados y para Yacuanquer, en marzo, siempre y cuando se cuente con buen drenaje para eliminar el exceso de agua, resultante de la mayor precipitación, presentada en esa época de siembra.

GRICAND402, GRICAND404 y GRICAND407, con promedios de rendimiento de 9,60, 8,93 y 9,92t.ha ${ }^{-1}$, respectivamente, que están por encima de la media general, presentaron un $\beta \neq 1$ y una desviación de la regresión $\mathrm{S}^{2}>0$ (Tabla 3, Figura 2), indicando que no son adaptables ni predecibles, por lo tanto, a pesar de alcanzar rendimientos promedios altos, no son recomendados para los ambientes evaluados. Finalmente, GRICAN401, con una media de $5,64 \mathrm{t} \cdot \mathrm{ha}^{-1}$, inferior a la media general, tampoco fue recomendable para los diferentes ambientes en estudio, debido a que, además de sus bajos rendimientos, no fue adaptable ni predecible. Los rendimiento alcanzados en la presente investigación concuerdan con los obtenidos por Khan et al. (2013), quienes evaluando genotipos de arveja arbustiva, reportando un rango entre 3,74 y 10,43t.ha ${ }^{-1}$.

Es importante resaltar que las líneas GRICAND405 adaptable a todos los ambientes y predecible y GRICAND406, adaptable a los mejores ambientes e igualmente predecible, son arvejas con el gen recesivo afila, el cual, se expresa remplazando las hojas laterales por zarcillos (Marx, 1987; Singh et al. 2013), permitiendo a las plantas mantenerse erectas al entrelazar sus zarcillos, evitando el acame o 
volcamiento, lo cual, reduce pérdidas en rendimiento y facilita las labores de cosecha. Además, las plantas con gen afila tienen una arquitectura, que permite incrementar la densidad de población, buscando mejorar los rendimientos.

Reacción a enfermedades causadas por Oídio y el complejo Ascochyta: En la época más lluviosa (ambientes de Yacuanquer marzo y Guaitarilla marzo), se evidenció la mayor presencia de las enfermedades $A$. pisi y $M$. pinodes, con una severidad en follaje de 55 a $75 \%$ y de 45 a $70 \%$, respectivamente y, en vainas, de 54 a $75 \%$, para $A$. pisi y de 10 a 25\%, para $M$. pinodes (Tabla 4). Lo anterior sugiere una reacción de las ocho líneas moderadamente susceptible a A. pisi, en follaje y vainas, moderadamente susceptibles a $M$. pinodes, en follaje, aunque la presencia de la enfermedad en vainas fue baja, sugiriendo resistencia, de acuerdo con la escala de evaluación. Es importante notar que el avance de la enfermedad para $A$. pisi, se dio desde la base hacia el ápice, mientras que en $M$. pinodes, se presentó de manera inversa o descendente, confirmando lo reportado por Tamayo (2000). En esta misma época, las condiciones de alta precipitación no favorecieron el ataque de Oídio (E. pisi).

Tabla 4. Niveles máximos alcanzados en follaje y vainas por los patógenos Oídio (Erisyphe pisi) y complejo Ascochyta (Ascochyta pisi y Mycosphaerella pinodes), en la evaluación de 8 líneas de arveja arbustiva, en seis ambientes de la zona cerealista de Nariño.

\begin{tabular}{|c|c|c|c|c|c|c|c|c|c|c|c|c|}
\hline \multicolumn{13}{|c|}{ Porcentaje de severidad de Erisyphe pisi. } \\
\hline Época & \multicolumn{4}{|c|}{ Época 1} & \multicolumn{4}{|c|}{ Época 2} & \multicolumn{4}{|c|}{ Época 3} \\
\hline Localidad & \multicolumn{2}{|c|}{ Yac } & \multicolumn{2}{|c|}{ Gua } & \multicolumn{2}{|c|}{ Yac } & \multicolumn{2}{|c|}{ Gua } & \multicolumn{2}{|c|}{ Yac } & \multicolumn{2}{|c|}{ Gua } \\
\hline Líneas & Fol & Vai & Fol & Vai & Fol & Vai & Fol & Vai & Fol & Vai & Fol & Vai \\
\hline GRICAND401 & 1 & 0 & 1 & 0 & 10 & 1 & 9 & 0 & 85 & 65 & 80 & 60 \\
\hline GRICAND402 & 1 & 0 & 0 & 0 & 0 & 0 & 1 & 0 & 10 & 0 & 8 & 0 \\
\hline GRICAND403 & 1 & 0 & 1 & 0 & 25 & 12 & 18 & 2 & 88 & 79 & 99 & 95 \\
\hline GRICAND404 & 2 & 0 & 3 & 0 & 85 & 36 & 52 & 15 & 93 & 86 & 99 & 90 \\
\hline GRICAND405 & 2 & 0 & 1 & 0 & 48 & 7 & 21 & 2 & 60 & 57 & 65 & 60 \\
\hline GRICAND406 & 5 & 1 & 3 & 0 & 48 & 5 & 46 & 8 & 90 & 85 & 99 & 90 \\
\hline GRICAND407 & 6 & 2 & 3 & 0 & 90 & 88 & 20 & 3 & 95 & 80 & 85 & 80 \\
\hline GRICAND408 & 4 & 2 & 1 & 0 & 88 & 15 & 47 & 37 & 95 & 88 & 99 & 97 \\
\hline \multicolumn{13}{|c|}{ Porcentaje de severidad de Ascochyta pisi. } \\
\hline GRICAND401 & 65 & 54 & 75 & 75 & 45 & 10 & 50 & 20 & 15 & 5 & 28 & 8 \\
\hline GRICAND402 & 70 & 65 & 75 & 68 & 55 & 15 & 58 & 20 & 20 & 5 & 36 & 8 \\
\hline GRICAND403 & 60 & 52 & 71 & 69 & 50 & 10 & 45 & 15 & 18 & 5 & 26 & 5 \\
\hline GRICAND404 & 66 & 72 & 53 & 63 & 35 & 20 & 40 & 25 & 25 & 8 & 28 & 6 \\
\hline GRICAND405 & 55 & 57 & 70 & 54 & 35 & 15 & 35 & 20 & 20 & 5 & 21 & 4 \\
\hline GRICAND406 & 60 & 55 & 75 & 55 & 30 & 20 & 30 & 30 & 25 & 5 & 30 & 10 \\
\hline GRICAND407 & 74 & 64 & 75 & 54 & 45 & 25 & 40 & 25 & 28 & 5 & 23 & 7 \\
\hline GRICAND408 & 58 & 54 & 65 & 55 & 45 & 25 & 49 & 30 & 20 & 10 & 22 & 9 \\
\hline \multicolumn{13}{|c|}{ Porcentaje de severidad de Mycosphaerella pinodes. } \\
\hline GRICAND401 & 67 & 14 & 70 & 10 & 43 & 13 & 49 & 11 & 12 & 1 & 11 & 1 \\
\hline GRICAND402 & 53 & 11 & 58 & 10 & 31 & 12 & 28 & 11 & 12 & 1 & 11 & 1 \\
\hline GRICAND403 & 54 & 12 & 60 & 20 & 42 & 13 & 35 & 13 & 12 & 1 & 11 & 3 \\
\hline GRICAND404 & 59 & 12 & 45 & 16 & 33 & 13 & 37 & 11 & 11 & 2 & 12 & 2 \\
\hline GRICAND405 & 49 & 13 & 50 & 12 & 31 & 12 & 38 & 11 & 12 & 2 & 11 & 3 \\
\hline GRICAND406 & 48 & 12 & 56 & 15 & 35 & 12 & 38 & 14 & 12 & 1 & 12 & 4 \\
\hline GRICAND407 & 65 & 15 & 69 & 25 & 45 & 15 & 43 & 11 & 13 & 2 & 12 & 1 \\
\hline GRICAND408 & 56 & 12 & 60 & 10 & 41 & 12 & 33 & 11 & 11 & 1 & 14 & 2 \\
\hline Grado: & \multicolumn{2}{|c|}{ 1:0-10\% } & \multicolumn{2}{|c|}{$2: 11-25 \%$} & \multicolumn{2}{|c|}{$3: 26-50 \%$} & \multicolumn{3}{|c|}{$4: 51-75 \%$} & \multicolumn{3}{|c|}{ 5:76-100\% } \\
\hline
\end{tabular}

Yac: Yacuanquer; Gua: Guaitarilla; Fol: Follaje; Vai: Vaina.

Los valores están dados en porcentaje. 
En los ambientes de Yacuanquer abril y Guaitarilla abril, al presentar una disminución en la precipitación durante el ciclo, se observó un menor ataque Ascochyta pisi en todas las líneas, con una severidad entre 30 a $58 \%$, en follaje y de 10 a 30\%, en vainas, mientras que para $M$. pinodes, se presentó una severidad entre 28 y $49 \%$, en follaje y de 11 a 15\%, en vainas. GRICAND405 y GRICAND406 mostraron una severidad de 30 y $35 \%$, respectivamente, en las condiciones climáticas de esta época, destacándose sobre las líneas restantes, que fueron más susceptibles. El patógeno E. pisi aumentó el porcentaje de severidad, en comparación con la siembra de marzo, alcanzando una severidad entre 85 a $90 \%$, en las líneas GRICAND404, GRICAND407 y GRICAND408; de 18 a 48\%, en GRICAND403, GRICAND405 y GRICAND406; GRICAND401 presentó una severidad entre 9 y 10\% y GRICAND402, menos del $1 \%$.

En la época de siembra más seca (ambientes de Yacuanquer mayo y Guaitarilla mayo), el complejo Ascochyta, se presentó con menor severidad, respecto a las siembras de marzo y abril. La severidad de A. pisi en follaje, para las ocho líneas evaluadas, estuvo entre $15 \mathrm{y}$ $30 \%$ y, en vainas, entre 5 y $10 \% ; M$. pinodes tuvo una severidad en follaje entre 11 al 14\% y en vainas, menor al 5\%. Los resultados sugieren que las condiciones de sequía, como las observadas en la siembra de mayo, no fueron favorables para el desarrollo de los patógenos del complejo Ascochyta y, en consecuencia, no fueron apropiadas para observar la reacción de las líneas a $A$. pisi y $M$. pinodes. En contraste, el patógeno E. pisi, se presentó con grados de mayor severidad respecto a las otras dos épocas, al encontrar una condición ambiental seca, con lloviznas frecuentes, que favoreció su desarrollo. En esta época (ambientes de siembra de mayo), se destacó la línea GRICAND402 que, para follaje, presentó entre 8 y $10 \%$ y, en vainas, no hubo afectación, sugiriendo una reacción de alta resistencia a Oídio, de acuerdo con la escala de evaluación, confirmando los resultados obtenidos por Checa et al. (2017), quienes consideraron esta línea como promisoria para futuras evaluaciones, por su reacción de resistencia al patógeno E. pisi.

GRICAND405 presentó un ataque intermedio, con una severidad entre 60 y 65\%, indicando una reacción moderadamente susceptible; en las demás líneas, se evidenció una reacción de susceptibilidad, alcanzando porcentajes de severidad, entre 80 y 99\%. Valencia et al. (2012), en un estudio de la reacción del complejo Ascochyta, en 20 líneas de arveja arbustiva, reportaron que los porcentajes de severidad de $A$. pisi y $M$. pinodes presentaron grados moderadamente susceptibles y moderadamente resistentes, con resultados similares a esta investigación. Según Tamayo (2000), los síntomas causados por $A$. pisi y $M$. pinodes, se presentan con mayor severidad en épocas lluviosas, lo cual, coincidió con la época de marzo, del presente estudio. El hongo E. pisi es más prevalente y severo en condiciones ambientales secas o durante periodos de verano prolongado.

En conclusión, las líneas de alto rendimiento y con comportamiento predecible fueron GRICAND405, con adaptación a todos los ambientes evaluados y GRICAND406, con mejor respuesta en los ambientes más favorables. Los mayores rendimientos, se obtuvieron en los ambientes de Yacuanquer abril, Guaitarilla abril y Yacuanquer marzo. Las líneas GRICAND404 y GRICAND402 sobresalieron en número de vainas por planta. Por otra parte, la época de siembra de marzo presentó la mayor severidad de ataque del complejo Ascochyta y los ambientes de la siembra de mayo, la mayor severidad de Oídio. La línea GRICAND402 presentó una reacción de alta resistencia a Oídio (Erysiphe pisi).

Agradecimientos: Los autores agradecen al Grupo de Cultivos Andinos de la Facultad de Ciencias Agrícolas de la Universidad de Nariño, por su apoyo en el establecimiento de los ensayos de campo; al Centro Internacional de Producción Limpia, Sena Lope Seccional Nariño, por permitir el desarrollo de esta investigación en sus instalaciones y a la I.A. Verónica Rosero, por su apoyo en la investigación. Conflicto de intereses: El manuscrito fue ejecutado, preparado y revisado con la participación de todos los autores, quienes declaramos que no existe conflicto de intereses que ponga en riesgo la validez de los resultados presentados. Financiación: Esta investigación fue financiada por Colciencias y la Universidad de Nariño.

\section{REFERENCIAS}

1. AULICINO, M.B.; LAOS, F.; ARTURI, M.J.; OROZCO, A.S.; GRECO, C. 2000. Análisis de la interacción genotipoambiente para rendimiento forrajero en cebadilla criolla. Invest. Agr. Prod. Prot. Veg. (España). 15(3):169-180.

2. BOLAÑOS, A. 2001. Introducción a la Olericultura. Ed. Universidad Estatal a Distancia (Costa Rica). 223p.

3. CAMARGO, D; ÁVILA, E. 2014. Efectos del Trichoderma sp. sobre el crecimiento y desarrollo de la arveja (Pisum sativum L.). Ciencia y Agricultura. (Colombia). 11(1):91-100.

4. CASANOVA, E.; SOLARTE, J.; CHECA, O. 2012. Evaluación de cuatro densidades de siembra en siete líneas promisorias de arveja arbustiva (Pisum sativum L.). Rev. Ciencias Agrícolas. (Colombia). 29(2):129-140.

5. CHECA, O.; BASTIDAS, J.; NARVAÉZ, C. 2017. Evaluación agronómica y económica de arveja arbustiva (Pisum sativum L.) en diferentes épocas de siembra y sistemas de tutorado. Rev. U.D.C.A Act. \& Div. Cient. (Colombia). 20(2):279-288. https://doi.org/10.31910/rudca.v20.n2.2017.380

6. CHECA, O.; RODRÍGUEZ, M. 2015. Resistencia a Oídio (Erysiphe polygoni) y rendimiento en arveja afila (Pisum sativum L.). Temas Agrarios. (Colombia). 20(2):58-71. https://doi. org/10.21897/rta.v20i2.759

7. EBERHART, S.; RUSSELL, W. 1966. Stability parameters for comparing varieties. Crop Science. (Estados Unidos). 6(1):36-40. https://doi.org/10.2135/cropsci1966.001118 3X000600010011x 
8. FEDERACIÓN NACIONAL DE CULTIVADORES DE CEREALES Y LEGUMINOSAS, FENALCE. 2016. Área, Producción y Rendimiento Cereales y Leguminosas, 2016 A. Disponible desde internet en: http:/ /www.fenalce.org/ nueva/plantillas/arch_down_load/2016_A_APR_junio. pdf (con acceso el 05/06/2016).

9. GONZÁLEZ, F.; LIGARRETO, G. 2006. Rendimiento de ocho genotipos promisorios de arveja arbustiva (Pisum sativum L.) bajo sistema de agricultura protegida. Fitotecnia Colombiana. 6(2):52-61.

10. HEDLEY, C.; AMBROSE, M. 1981. Designing “leafless" plants for improving the dried pea crop. Advances in Agronomy. (Estados Unidos). 34:225-227.

11. INSTITUTO DE HIDROLOGÍA, METEOROLOGÍA Y ESTUDIOS AMBIENTALES DE COLOMBIA, IDEAM. 2018. Departamento Administrativo de Estadística. Disponible desde internet en: www.ideam.gov.co (con acceso el 15/03/2018).

12. INSTITUTO GEOGRÁFICO AGUSTÍN CODAZZI, IGAC. 2004. Estudio General de suelos y clasificación de tierras del Departamento de Nariño. Subdirección de Agrología (Bogotá D. C.). 733p.

13. JANNINK, J.L.; LIEBMAN, M.; MERRICK, L.C. 1996. Biomass production and nitrogen accumulation in pea, oat and vetch green manure mixtures. Agron. J. (Estados Unidos). 88(2):231-240.

14. KHAN, T.; RAMZAN, A.; JILLANI, G.; MEHMOOD, T. 2013. Morphological performance of peas (Pisum sativum) genotypes under rainfed conditions of Potowar región. J. Agric. Res. (Estados Unidos). 51(1):51-60.

15. KUJALA, V. 1953. Felderbse, bei welcher die ganze Blattspreite in Ranken umgewandelt ist. Arch. Soc. Zool. Bot. Fenn. (Finlandia). 8:44-45.

16. LigARretTO, G.; CASTRO, O.; CHÁVES, B. 2015. Estabilidad fenotípica de una colección de fríjol andino (Phaseolus vulgaris L.) tipo arbustivo. Rev. U.D.C.A Act. \& Div. Cient. (Colombia). 18(1):109-118.

17. MARX, G. 1987. A suite of mutants that modify pattern formation in pea leaves. Plant Mol. Biol. Rep. (Estados Unidos). 5:311-335.

18. MiHAILOVIC, V. 2008. Componentes de rendimiento de grano afila (af) líneas de guisantes forrajeros (Pisum sativum L.). Instituto de Cultivos y Hortalizas (Serbia). 98p.

19. MORA, F.; OSMÉRIO, P.J.; SCAPIM, C.A. 2007. Predicción del efecto de cultivares de algodón en la presencia de interacción genotipo-ambiente. Cien. Inv. Agr. (Chile). 34(1):13-21.

20. PACHECO, C.; VERGARA, M.; LIGARRETO, A. 2011. Clasificación de 42 líneas mejoradas de arveja (Pisum sativum L.) por caracteres morfológicos y comportamiento agronómico. Rev. Facultad Nacional de Agronomía Medellín. 63(2):5543-5553.

21. PROHENS, J.; NUEZ, T. 2007. Vegetables II: Liliaceae, Solanaceae, and Umbelliferae. Springer Science and Bussines Media. Handbook of plant breeding. Springer. (New York). 365p.

22. SAÑUdO, B.; CHECA, O.; ARTEAGA, G. 1999. Manejo agronómico de leguminosas en zonas cerealistas - UdenarProfriza Corpoica-Fenalce-Corpotrigo. (Pasto). 97p.

23. SIDDIQUE, A.; WRIGHT, D.; MAHBUB ALI, S. 2002. Effects of sowing dates on the phenology, seed yield and yield components of peas. J. Biological Sciences. (Estados Unidos). 2(5):300-303. https://doi.org/10.3923/ jbs. 2002.300 .303

24. SINGH, M.; HARI, D.; BISTH, S. 2013. Genetic and genomic resources of grain legume improvement. Ed. First edition. Elsevier Insights. (London). 322p.

25. SUMMERFIELD, R.; ROBERTS, E.; ELLIS, R.; LAWN, R. 1991. Towards the reliable prediction of time to flowering in six annual crops. I. The development of simple models for fluctuating field environments. Exp. Agric. (Brasil). 27(1):11-31. https://doi.org/10.1017/S0014479700019165

26. TAMAYO, J. 2000. Enfermedades del cultivo de arveja en Colombia: Guía de reconocimiento y control. Boletín técnico. Fondo Nacional de Leguminosas. (Colombia). 50p.

27. VALENCIA, A.; TIMANA, Y.; CHECA, O. 2012. Evaluación del complejo Ascochyta Ascochyta pisi y Mycosphaerella pinodes (A. pinodes) en 20 líneas de arveja (Pisum sativum L.). Rev. Ciencias Agrícolas. (Colombia). 29(2):39-52.

28. VARGAS, E.; VARGAS, J.; BAENA, D. 2016. Análisis de estabilidad y adaptabilidad de híbridos de maíz de alta calidad proteica en diferentes zonas Agroecológicas de Colombia. Acta Agron. 65(1):72-79. http://doi. org/10.15446/acag.v65n1.43417

29. VÁSQUES, V.; CABRERA, H.; JIMÉNEZ, L.; COLUNQUE, A. 2019. Estabilidad del rendimiento de genotipos de papa (Solanum tuberosum L.). Ecol. Apl. (Perú). 18(1):59-65.

30. WANG, F.; FU, J.; DONG, L.; ZHU, Y. 2003. Tendril inheritance in semi-leafless pea and its utilization in breeding. YiChuan $=$ Hereditas (China). 25(2):185-188. 